\title{
Neutrophil extracellular traps in cancer progression
}

\author{
Jonathan Cools-Lartigue $\cdot$ Jonathan Spicer $\cdot$ \\ Sara Najmeh · Lorenzo Ferri
}

Received: 22 April 2014/Revised: 22 June 2014 / Accepted: 14 July 2014/Published online: 29 July 2014

(C) Springer Basel 2014

\begin{abstract}
Neutrophils are being increasingly recognized as an important element in tumor progression. They have been shown to exert important effects at nearly every stage of tumor progression with a number of studies demonstrating that their presence is critical to tumor development. Novel aspects of neutrophil biology have recently been elucidated and its contribution to tumorigenesis is only beginning to be appreciated. Neutrophil extracellular traps (NETs) are neutrophil-derived structures composed of DNA decorated with antimicrobial peptides. They have been shown to trap and kill microorganisms, playing a critical role in host defense. However, their contribution to tumor development and metastasis has recently been demonstrated in a number of studies highlighting NETs as a potentially important therapeutic target. Here, studies implicating NETs as facilitators of tumor progression and metastasis are reviewed. In addition, potential mechanisms by which NETs may exert these effects are explored. Finally, the ability to target NETs therapeutically in human neoplastic disease is highlighted.
\end{abstract}

Keywords Neutrophil extracellular traps - Neutrophil · Cancer $\cdot$ Metastatis · Inflammation

\section{Introduction}

Inflammation is described as one of the hallmarks of cancer; whereby inflammatory cells and the factors they elaborate have been implicated in nearly every step of tumor development and progression [1]. Neutrophils have

J. Cools-Lartigue $(\bowtie) \cdot$ J. Spicer $\cdot$ S. Najmeh $\cdot$ L. Ferri

LD MacLean Surgical Research Laboratories, Department of Surgery, McGill University, Montreal, QC, Canada

e-mail: jonathan.cools-lartigue@mail.mcgill.ca been increasingly recognized as important components of the tumor-associated inflammatory cell infiltrate [2-5]. Accordingly, their role in tumor biology is being increasingly recognized. In fact, both pro- and antitumorigenic properties have been demonstrated [6,7]. It is important to note that the term "neutrophil" is a descriptive term based on the histologic appearance of a circulating leukocyte [8]. In clinical practice, the term neutrophil denotes a homogenous population of cells that are mobilized in response to inflammatory stimuli [8]. However, this term does not account for the diversity in cellular functions observed in the laboratory setting. Thus, while circulating neutrophils are morphologically similar under light microscope, it is likely that they can harbor or adopt distinct genetic expression profiles suited to a particular effector mechanism [6, 7, 9]. Along these lines, distinct subsets of "neutrophils" have been described that exert specific and sometimes contradictory roles in tumor development [5-7].

Most recently, neutrophil extracellular traps (NETs) have been recognized as an important antimicrobial effector mechanism elaborated by neutrophils in response to numerous stimuli. Recent evidence has expanded their biologic scope, with a variety of studies implicating NETs in tumor biology. These range from tumor-associated thrombosis to tumor progression. While these findings are novel, it is important to understand that NETs are complex structures composed of a chromatin decorated in neutrophil-derived peptides, the breadth of which have yet to be fully elucidated. However, many of these proteins have been rigorously studied in the field of cancer progression, albeit in an entirely NET-independent context. In the present review, we will focus on the newly described role of NETs as potential promoters of metastasis. In addition, the contribution of individual NET-associated proteins to tumor progression will be reviewed. 


\section{Neutrophil extracellular traps}

Recently, NETs have been recognized as an additional arm to the antimicrobial armamentarium of neutrophils. Under appropriate stimuli, neutrophils are able to extrude their nuclear DNA, resulting in its web-like projection into the extracellular environment. This DNA is decorated in modified histones and antimicrobial peptides and has been shown to trap and kill pathogens both in vitro and in vivo [10-16].

In response to infection, NETs appear to increase the efficiency of bacterial trapping beyond what is possible by receptor-mediated endocytosis alone [17]. This has recently been demonstrated in an in vivo model of Escherichia coli and Staphylococcus aureus infection in mice [17]. In the study by McDonald et al., the authors demonstrate that bacterial trapping within the sinusoidal spaces by resident Kupffer cells is unchanged in mice stimulated to produce intravascular NETs versus untreated mice. This was taken to suggest that the receptor-mediated mechanism of leukocyte-pathogen interaction is rapidly overwhelmed in the context of infection. However, NETs provide neutrophils with the ability of interacting with pathogens across a large surface area [17].

NETs have been shown to mediate trapping of both Gram-positive and Gram-negative bacteria, fungal, protozoan and even viral invaders [11]. Multiple consequences to trapping have been observed. Direct bacterial killing has been observed in both Shigella flexnerii and S. aureus [15]. This is thought to be mediated by highly toxic modified histones [15, 17]. In addition, inactivation of bacterial virulence factors has been observed and directly attributed to the action of neutrophil elastase [12]. Finally, the trapping or immobilizing role of NETs is thought to increase the efficiency of subsequent phagocytosis by additional leukocytes [17, 18].

Numerous stimuli have been shown to result in NET formation. These include exposure of primed neutrophils to bacterial products such as lipopolysaccharide (LPS), formyl-methionyl-leucyl-phenylalanine (fMLP), phorbol esters such as phorbol myristate acetate (PMA) and physical interaction with activated platelets $[15,17-20]$. Additional cytokines, such as tumor necrosis factor alpha (TNF- $\alpha$ ) and interleukin 8 (IL-8), have been shown to facilitate the process of NET formation. In addition, both of these cytokines are elaborated by a number of primary tumor types [21-23]. Despite this fact, studies to date have not definitively demonstrated NET formation in the context of cancer secondary to the production of these cytokines.

During the process of NET formation, characteristic morphologic changes within neutrophils can be observed $[15,18,24]$. Shortly after stimulation, finger-like projections at the cell membrane can be observed, with subsequent flattening of cells [24]. Chromatin decondensation is a prominent feature of NET formation and is associated with histone modification. Citrullination of histone $\mathrm{H} 3$ by peptidylarginine deiminase 4 (PAD 4) is important during the process of NETosis, with its inhibition greatly reducing the efficiency of this process $[25,26]$. Following chromatin decondensation, loss of nuclear morphology and loss of the nuclear membrane are observed and are prominent features of NETosis [24]. These are followed by the eventual extrusion of DNA from the cell [24]. This process is dependent on the generation of reactive oxygen species (ROS), as evidenced by the fact that patients with chronic granulomatous disease demonstrate reduced NET formation [12, 13, 27]. In addition, activation of the mitogen-activated protein (MAP) kinase pathway is essential [28]. Following activation of the appropriate signaling cascades, DNA extrusion ultimately occurs, which requires the translocation of neutrophil elastase (NE) to the nucleus [29]. This process is potentiated by the activity of myeloperoxidase (MPO) [29, 30]. Neutrophil elastase plays an essential role in the extrusion of neutrophil-derived nuclear DNA into the extracellular environment during NET formation. Following NE inhibition with a neutrophil elastase inhibitor (NEi) or serum leukocyte protease inhibitor (SLPI), chromatin decondensation, nuclear degranulation, and consequently neutrophil death and NETosis are disrupted [29].

Structural evaluation of NETs by both light and electron microscopy has demonstrated that they are composed of a filamentous DNA backbone [15]. Electron microscopy demonstrates $17 \mathrm{~nm}$ fibers, which can interact with one another to form significantly thicker fibers of up to $50 \mathrm{~nm}$ [15]. These DNA projections are decorated by a number of granule proteins $[14,16,20]$. These include NE, MPO, calprotectin, cathepsin $\mathrm{G}(\mathrm{CG})$, proteinase 3 (PR3), matrix metalloproteinase 9 (MMP-9) and bactericidal/permeability-increasing protein (BPI) [14, 16, 20]. Most of these molecules have been shown to participate in both direct and indirect pathogen-killing mechanism and are thus thought to play an important role in the microbicidal activity of NETs [31].

\section{Neutrophil extracellular traps at the primary tumor}

The role that NETs play in tumor progression has only started to be characterized and remains poorly understood. While few studies have been performed to date, evidence does suggest a potential association between intra-tumoral NET deposition and tumor progression in both experimental models and in human cancer patients [32-34]. Zychlinsky et al. assessed surgical resection specimens from eight patients with Ewing sarcoma for the presence of 
tumor-associated neutrophils (TAN) and NETs as determined by positive staining for extracellular MPO [33]. Of these eight patients, two $(25 \%)$ demonstrated intra-tumor NET deposition. Post-neoadjuvant chemotherapy and surgery, these two patients developed early relapse, although the site was not specified in the study [33]. These observations lead the authors to hypothesize that Ewing sarcoma cells can stimulate TAN to release NETs, which may serve as a marker of poor prognosis [33].

Additional evidence in the literature supports this hypothesis. In the study by Ho-Tin-Noé et al., large numbers of neutrophils and extracellular chromatin were observed in 9-day-old LLC tumors. While the authors never explicitly state that these structures represent NETs, the presence of hypercitrullinated neutrophils and extracellular chromatin bearing this histone modification is strong evidence in support of this identity and is in keeping with the results of Zychlinsky et al. in Ewing sarcoma [35].

The ability of tumor cells to predispose neutrophils to produce NETs has been demonstrated in a number of tumor types. This further highlights the possibility that NETs play an important role in tumor biology and further support the human data published to date. Demers et al. [36] demonstrated that multiple tumor types including hematologic, mammary and lung neoplasms, are able to predispose circulating neutrophils to produce NETs. The authors demonstrate this phenomenon in a variety of ways. First, they generated mice with a chronic myelogenous leukemia (CML)-like myleoproliferative neoplasm, accomplished by bone marrow engraftment of cells expressing the $t(9 ; 22)$ breakpoint cluster region-Abelson (BCR-ABL-1) translocation [36]. This results in the constitutive activation of the Abelson murine leukemia viral oncogene homolog 1 (ABL1)-encoded tyrosine kinase, leading to unregulated cellular proliferation associated with CML in humans [36]. These mice were found to express significantly higher quantities of plasma cell-free DNA (cfDNA) compared to control mice [36]. Isolation of circulating neutrophils followed by stimulation with platelet-activating factor (PAF) revealed that neutrophils derived from CML-like mice were more prone to NET formation compared to neutrophils from wild-type mice [36]. These findings support the hypothesis that primary tumors can facilitate NET production from circulating neutrophils.

The authors subsequently go on to demonstrate that this phenomenon is not unique to the CML model employed [36]. Injection of $4 \mathrm{~T} 1$ mammary carcinoma cells into the mammary fat pad of mice results in a time-dependent increase in circulating neutrophils and cfDNA [36]. This cell-free DNA likely represented NETs as evidenced by the observation of a time-dependent increase in the number of circulating neutrophils bearing a hypercitrullinated histone H3 up until day 28 [36]. Histone citrullination, particularly histone $\mathrm{H} 3$, plays an important role in NET elaboration and is thought to be specific to this process [36]. Following day 28 , a reduction in the number of these neutrophils was observed with a concomitant rise in plasma levels of citrullinated $\mathrm{H} 3$ [36].

As in the previous experiment, neutrophils isolated from tumor-bearing mice produced more NETs in response to a given dose of exogenous PAF compared to neutrophils isolated from healthy control mice [36]. Furthermore, this effect is most pronounced with neutrophils isolated from tumor-bearing mice after 14 days [36]. The authors made similar observations in Lewis lung carcinoma (LLC) tumor-bearing mice, further suggesting that this phenomenon may not be unique to a particular tumor type [36].

This effect was attributed to granulocyte-colony stimulating factor (G-CSF) production by tumors. Antibodymediated neutralization of serum G-CSF inhibited neutrophilia and reduced the sensitivity of neutrophils to PAF [36]. Taken together, these data appear to confirm the hypothesis that tumors are able to promote NET formation from host neutrophils [36].

The studies by Demers and Ho-Tin-Noé et al. suggest that NET deposition in response to neoplastic cells begins to take place at approximately day 9-14 following tumor implantation. This time frame may be of importance as the effect of neutrophil ablation on primary tumor growth changes over time. Mishalian et al. demonstrated diminished growth of LLC and AB12 mesothelioma tumors following neutrophil depletion at day 14 post-implantation. Conversely, ablation of neutrophils prior to day 7 failed to inhibit their growth. A more detailed examination of infiltrating neutrophils demonstrated that early on during tumor development, they localized to the tumor margins and could not be seen infiltrating to the same extent as to what was observed after day 14 . More significantly, these neutrophils appeared to be functionally distinct, with intratumoral neutrophils identified at the later stage of development demonstrating a more pro-tumoral phenotype than those identified at the tumor periphery in early stages [37]. These observations are fascinating as they demonstrate a temporal link between tumor infiltration by neutrophils, the elaboration of NETs and their contribution to tumor development.

The studies outlined thus far suggest that NET formation is induced within the primary tumor by a number of neoplasms. Given the redundancy of this finding across a number of tumor types, it is feasible that intra-tumoral NET deposition confers some type of advantage to the neoplasm in question. Evidence has been put forward to date that supports this hypothesis. In the study by Sangaletti et al. [34], B cell proliferation and malignant transformation in the context of autoimmune disease were enhanced by NET formation. With regard to proliferation, 
the authors demonstrate that following stimulation of neutrophils with PMA to induce NET formation, co-culture with $\mathrm{B}$ cells results in a significant increase in proliferation [34]. The same observation was made when B cells were co-cultured with murine neutrophils derived from autoimmune mice [34]. These leukocytes were prone to undergo spontaneous NETosis, suggesting that this was responsible for enhanced B cell proliferation [34]. In further support of this hypothesis, the authors demonstrated that the addition of DNAse to neutrophils undergoing NETosis abolished their proliferative effect on B cells [34]. This effect was found to occur secondary to increased nuclear factor kappa-light-chain enhancer of activated B cells (NFKB) signaling [34]. In vivo, the authors demonstrate enhanced staining with $\mathrm{Ki}-67$, a marker of proliferation, in B cells within autoimmune lymphoid follicles in addition to enhanced expression of B cell lymphoma 2 (Bcl-2) [34]. Taken together, these results support a direct proliferative role for NETs on B cells providing a proof of concept that NETs may directly influence tumor cell behavior in support of ongoing growth and development.

Collectively, the data presented thus far demonstrate that NET formation occurs within the primary tumor and is associated with adverse clinical outcomes in human disease. At the experimental level, it appears that neutrophil infiltration of the primary tumor is associated with enhanced growth. Several studies have demonstrated enhanced neoplastic cell survival and migration as a result of local tumor-neutrophil interactions. Dimitru et al. demonstrated in a head and neck cancer (HNC) model that tumor-infiltrating neutrophils were able to enhance tumor cell survival and migration through the upregulation of their proinflammatory functions, more specifically by the release of chemokine $\mathrm{C}-\mathrm{C}$ motif ligand-4 (CCL4), chemokine $\mathrm{C}-\mathrm{X}-\mathrm{C}$ motif ligand 8 (CXCL8, also known as IL8) and MMP-9 [46]. In another study by Acharyya et al., a population of breast cancer cells that overexpressed CXCL1/2 were able to attract Gr1+/CD11b+ neutrophil precursor cells into the tumor. These granulocytes were shown to increase local tumor survival through the secretion of chemokines, Including S100A8/A9 [47]. Bald et al. investigated the mechanisms underlying the increase in metastatic progression of primary cutaneous melanoma following repeated ultraviolet exposure. The authors demonstrated the recruitment of neutrophils and a subsequent inflammatory cascade as a result of UV exposure. This inflammatory response was shown to promote local angiogenesis and migration of melanoma cells toward endothelial cells. These interactions resulted in perivascular invasion, a phenomenon termed "angiotropism" [48]. In addition, NETs themselves appear to facilitate primary tumor development through both the inhibition of apoptosis and via a direct proliferative effect [33-37]. Given these findings, the distinct possibility that NETs promote tumor progression, ultimately leading to metastasis arises.

Potential contribution of NET components to tumor progression

The data presented thus far suggest that NETs may act within the primary tumor to promote tumor progression. However, no studies to date have demonstrated the mechanism(s) underlying these observations. This being said, a tremendous body of literature on the role of specific neutrophil-derived proteins has been published to date. As previously stated, NETs are composed of neutrophilderived chromatin decorated with antimicrobial proteins and peptides. These include MMP-9, CG and NE whose role in tumor progression has been examined without specific reference to NETs being made. It is important to note that NETs are thought to provide a microenvironment that traps pathogens and brings them into close proximity with antimicrobial peptides, thus favoring their elimination. As a result, it is possible that in the context of malignancy NETs play an analogous role, whereby tumor cells are exposed to a high local concentration of biologically active proteins, favoring interactions that may act to promote proliferation, inhibit apoptosis and support egress from the primary tumor entirely. Accordingly, the literature outlining the contribution of the NET components MMP-9, $\mathrm{CG}$ and NE is worth discussing.

\section{Matrix metalloproteinase 9}

MMP-9 is released by neutrophils during degranulation and is postulated to assist in the process of extravasation via the degradation of the extracellular matrix (ECM) [16]. It has been implicated in a variety of pro-tumorigenic processes at nearly every step of the metastatic cascade [38-42]. These include proliferation, reduced apoptosis, increased angiogenesis, invasion and metastasis [38-42].

In a study by Coussens et al. [40], the authors employ a model of human papilloma virus (HPV) HPV-16 induced skin carcinogenesis in mice. They demonstrate a decreased frequency of invasive tumor development in MMP-9 -/mice compared to wild type (WT) [40]. This was associated with decreased keratinocyte proliferation in MMP-9 -/- mice compared to WT [40]. This phenotype was reversed by transplantation of WT BM cells in MMP-9 $-/-$ mice following irradiation suggesting that the source for MMP-9 is infiltrating bone marrow-derived cells [40]. This result is further supported by immunohistochemical analysis of squamous cell carcinoma (SCC), which revealed that MMP-9 positivity was restricted to invading granulocytes as opposed to tumor cells themselves [40]. Thus, the authors demonstrate that granulocyte-derived 
MMP-9 is associated with increased proliferation of neoplastic cells [40].

In addition to supporting tumor growth via increased proliferation, MMP-9 may also act to inhibit apoptosis in tumor cells [38]. In the study by Acuff et al. the authors demonstrate an $81 \%$ increase in the number of gross pulmonary metastases following tail vein injection of LLC cells in C57/B16 mice compared to MMP-9 -/- mice [38]. In keeping with this finding, the authors also demonstrated an approximate $50 \%$ decrease in the number of pulmonary nodules formed in MMP-9 -/- mice following orthotopic administration of A549 lung carcinoma cells [38].

As in the previous study, reconstitution of MMP-9 positive bone marrow-derived cells in MMP-9 -/- mice via bone marrow transplant rescued the metastatic phenotype observed in WT mice following tail vein injection of LLC cells [38]. The number of pulmonary metastases observed in this model in MMP null mice was $14 \%$ that of WT mice [38]. Thus, as in the study by Coussens et al., these authors demonstrate that leukocyte-derived MMP-9 supports tumor development [38, 40]. Furthermore, microscopic analysis revealed that tumor-infiltrating neutrophils appeared to be the predominant source of MMP-9 [38]. However, as per Acuff et al. [38], increased lung tumor growth was associated with a significantly lower rate of apoptosis in WT compared to MMP-9 -/- mice at early stages of tumor development. Following tail vein injection of fluorescently labeled A549 cells, apoptosis within the lungs was quantified. At $6 \mathrm{~h}$ post-injection, significantly more tumor cells underwent apoptosis in MMP-9 -/compared to WT mice ( 8 vs. $2 \%$ ). By $20 \mathrm{~h}$ post-injection the trend had reversed, which the authors attributed to the then insufficient tumor cells in the MMP-9 -/- mice to match that observed in WT mice [38]. Collectively, these results support the hypothesis that neutrophil-derived MMP-9 supports tumor growth in part through preventing apoptosis [38].

Neutrophil-derived MMP-9 has also been implicated in angiogenesis, which is required to maintain both primary and metastatic tumor growth [39, 41-43]. In a study by Van Coillie et al. [43], transgenic murine melanomas overexpressing granulocyte chemotactic peptide 2 (GCP2 ), a potent murine neutrophil-attracting chemokine, were generated and injected into nude mice. Compared to wildtype tumors, GCP-2 overexpressing tumors demonstrated increased growth, with significantly higher tumor volumes exhibited at 8 weeks following subcutaneous administration [43]. Conversely, no difference in growth was observed in vitro between transgenic and WT tumors [43]. This finding suggests that enhanced tumor growth in transgenic tumors is the result of interactions with hostderived cells [43]. Accordingly, immunohistochemical analysis of excised GCP-2 overexpressing tumors revealed well-developed vascular networks when compared with their WT counterparts [43]. Furthermore, transgenic tumors exhibited extensive infiltration with cells staining positive for MPO, which were interpreted as neutrophils [43]. This neutrophil infiltrate was associated with significantly increased levels of intra-tumoral MMP-9 when compared with WT tumors [43].

Additional evidence implicating neutrophils and neutrophil-derived MMP-9 as drivers of angiogenesis exists [39, 41-43]. Masson et al. [41] implanted malignant keratinocytes grown on collage gels subcutaneously into mice. Three weeks following implantation, vascular structures could be visualized penetrating through the collagen gel and into the overlying carcinoma cells, which demonstrated extensive growth and invasion [41]. Staining for MMP-9 demonstrated its co-localization with host neutrophils and not with ingrowing endothelial or tumor cells [41]. MMP-9 -/-, particularly in conjunction with MMP-2 -/-, was associated with profoundly impaired angiogenesis and tumor invasion despite that tumors were derived from MMP-9 WT cells, thus highlighting the importance of stromal cells [41].

In keeping with these observations, Bergers et al. [39] similarly demonstrated that infiltrating neutrophils were an important source of MMP-9, the elaboration of which is strongly associated with angiogenesis and tumor growth. The authors employed a model of pancreatic islet cancer, in which mice spontaneously form dysplastic lesions, which progress to carcinomas by 12-14 weeks of age [39]. During progression, dysplastic lesions exhibit enhanced angiogenesis as they develop into carcinomas [39]. This transition in phenotype has been termed the "angiogenic switch" [39, 42]. Using this model, the authors demonstrated dense populations of infiltrating neutrophils within dysplastic lesions undergoing angiogenesis [39]. Staining for MMP-9 revealed that its source was infiltrating neutrophils as opposed to neoplastic cells [39]. Infiltrating neutrophils were similarly observed in lesions that had progressed to carcinomas [39]. This observation suggested that MMP-9 expression by infiltrating neutrophils supported tumor development, possibly through facilitating the angiogenic switch [39]. Accordingly, neutrophil depletion with anti-GR1 antibodies resulted in a $57 \%$ reduction in the number of angiogenic lesions, if depletion occurred early in tumor development during the hyperplastic/ dysplastic phase [39]. Conversely, depletion of neutrophils at a later time point (11 weeks of age) did not alter tumor number or size [39]. These data suggest that neutrophil infiltration and the concomitant expression of MMP-9 are important in instigating angiogenic switch [39]. Although this is demonstrated within the primary tumor, the concept of MMP-9-mediated angiogenesis 
could be at play in a similar fashion within budding metastases. In this manner, NETs could act to support tumor development at distant organ sites. Note that antiGR1 is not neutrophil specific, as it can bind to the Ly6C receptor, which is also found on dendritic cells and subsets of monocytes, macrophages and lymphocytes, and can therefore eliminate these cells in addition to neutrophils [44]. As shown in literature, it has however been extensively used to deplete neutrophils in murine disease models [45-50] and the above-mentioned study is among this extensive body of literature. In an attempt to rectify these potential problems, additional studies have employed anti-GR1 Ly6G antibodies that demonstrate enhanced specificity to the granulocyte lineage, of which neutrophils are the most abundant [44, $51,52]$.

Neutrophil-derived MMP-9 appears to exert its effects on angiogenesis at least in part by liberating vascular endothelial growth factor (VEGF) from the ECM [39, 42]. Ablation of neutrophils in dysplastic islet lesions is associated with a reduction in VEGF:VEGFR co-localization by a factor of approximately $90 \%$ [39, 42]. Inhibition of MMP-9 with the specific small molecule inhibitor R94138 resulted in a decrease in the frequency of angiogenic switch and a consequent $70-80 \%$ reduction in the number of tumors [39, 42]. This implicates neutrophil-derived MMP-9 as the mediator of the angiogenic switch in this particular model [39, 42]. In addition, exogenous MMP-9 was able to render non-angiogenic islet lesions into angiogenic ex vivo. This effect was reduced by administration of anti-VEGF antibodies and could be replicated by the addition of heparanase, suggesting MMP-9 acts by liberating VEGF from the ECM via its proteolytic activity $[39,42]$.

\section{Cathepsin G}

Cathepsin G is a peptidase stored within azurophilic granules [53, 54]. It has demonstrated functions both in the degradation of engulfed bacteria and in ECM remodeling [53-58]. In addition, cathepsin $G$ has been shown to facilitate angiogenesis and tumor cell dissemination, potentially favoring the development of metastasis [59-62].

As tumor cells emigrate from the primary tumor site, the formation of tumor cell aggregates through homotypic cell-cell interactions has been observed [59, 60]. It is postulated that these aggregates disseminate in the bloodstream and form tumor emboli at distant sites, potentially leading to the establishment of metastatic foci $[59,60]$. Cathepsin $\mathrm{G}$ has been shown to facilitate the formation of tumor aggregates in in vitro models of breast cancer [59, 60]. Yui et al. [60] demonstrated that human MCF-7 mammary adenocarcinoma cell lines spontaneously formed spherical cell aggregates in the presence of neutrophils or following culture in the presence of activated neutrophil supernatant. Aggregation was mediated by intercellular adhesion via E-cadherin [60]. Neutrophil-derived cathepsin $G$ was found to be partially responsible for this aggregating phenotype, as exogenous addition of recombinant cathepsin G to MCF-7 cells in culture induced aggregation [60]. Furthermore, in the presence of activated neutrophil supernatant, inhibition of cathepsin $G$ abolished the aggregation of MCF-7 cells [60]. A similar study from the same group demonstrated that tumor cell aggregation was mediated by binding of cathepsin $\mathrm{G}$ to the cell surface and required its enzymatic activity [59].

In addition to the in vitro evidence presented above, in vivo evidence implicating cathepsin $\mathrm{G}$ in the formation of metastases exists. In human lung cancers, elevated levels of cathepsin $\mathrm{G}$ have been correlated with tumor grade and stage [63]. The source of cathepsin $G$ in these tissues was localized to tumor-infiltrating neutrophils as determined by immunohistochemistry [63]. Cathepsin $\mathrm{G}$ has been shown to facilitate the formation of osteolytic bone lesions in models of breast cancer metastasis [61, 62]. Wilson et al. [62] demonstrated elevated levels of cathepsin $\mathrm{G}$ at the tumorbone interface of murine breast cancer cells implanted into mouse calvarium. Bone lesion formation was mediated by increased osteoclastogenesis as a result of elevated cathepsin $\mathrm{G}$ production, induced by tumor cells. Inhibition of cathepsin $G$ using the small molecule inhibitor TCPK resulted in diminished bony lesion formation [62].

Once tumor cells have seeded a distant organ, ongoing tumor growth will ultimately require the ingrowth of new blood vessels [1, 64]. Along these lines, an additional study by Wilson et al. [62] demonstrated that cathepsin G might favor angiogenesis within osteolytic bony metastases in a murine model of breast cancer metastasis. Using a model of intra-calvarial tumor cell injection, the authors demonstrated reduced angiogenesis following administration of an exogenous cathepsin G inhibitor (TPCK) as determined by quantification of micro-vessel density [62]. This reduction in endothelial ingrowth was associated with reduced expression of both VEGF mRNA and protein [62]. Collectively, the evidence suggests that cathepsin G may act to facilitate ECM remodeling, favoring tumor progression and potentially metastasis in both experimental models and human disease [62].

\section{Neutrophil elastase}

Neutrophil elastase is released from azurophilic granules during neutrophil degranulation. It has a broad spectrum of activity ranging from the proteolytic activation of neutrophil-derived antibacterial proteins to the degradation of ECM components including elastin [57, 58, 65-68]. In 
addition to its postulated role in microbial defense and neutrophil trafficking, NE has demonstrated a variety of pro-tumorigenic roles, both in vitro and in vivo [69-76].

In vitro, NE has demonstrated the ability to directly enhance tumor cell proliferation and migration [70, 76]. Co-culture of polymorphonuclear leukocytes (PMN) with A549 cells resulted in increased proliferation of this tumor cell line. However, this effect was abrogated if co-culture experiments were performed using NE $-/-$ PMN. In keeping with this observation, proliferation of A549 cells in the presence of WT PMN was attenuated in the presence of an NE inhibitor [76]. Furthermore, addition of exogenous NE produced a similar, albeit more modest increase in proliferation at concentrations from 40 to $80 \mathrm{nM}$ [76]. This concentration of NE is similar to what has been demonstrated near the PMN cell surface following activation, further supporting the hypothesis of a direct proliferative role for NE on tumor cells [76].

The proliferative effects of NE on A549 cells were subsequently found to be mediated by activation of the phosphatidylinositol-4,5-bisphosphate 3-kinase (PI3 K) pathway [76]. Activation of PI3K was necessary for proliferation as evidenced by the fact that NE-mediated proliferation of A549 cells is attenuated in the presence of the specific PI3K inhibitors [76]. Furthermore, this was dependent on internalization of NE into endosomes within A549 cells $[70,76]$. This was found to lead to degradation of IRS-1 within the cytoplasm ultimately leading to PI3K activation and increased proliferation [70, 76].

Similar observations have been made in esophageal cancer in addition to lung cancer [73]. Wada et al. [73] demonstrated a dose-dependent increase in the proliferation of four separate esophageal cancer cell lines following addition of exogenous NE. This was inhibited by the addition of the NE inhibitor sivelestat [73]. Addition of NE was accompanied by an increase in concentrations of transforming growth factor $\alpha$ (TGF- $\alpha$ ), VEGF and plateletderived growth factor (PDGF) within the tumor cell culture supernatant, suggesting that NE may act by liberating these factors into the extracellular environment, potentially favoring interactions with their cognate receptors [73]. Along these lines, an additional study by the same authors demonstrated epidermal growth factor receptor (EGFR)mediated signaling as a direct consequence of the effects of $\mathrm{NE}$ on gastric carcinoma cell lines in vitro [75]. As with the esophageal cell lines, this translated into enhanced proliferation, which was abrogated by sivelestat [75]. Again, this was mediated by the proteolytic liberation of TGF- $\alpha$ from the cell surface [75]. Nawa et al. [77] demonstrated similar effects of NE and concomitant TGF- $\alpha$ signaling in an in vitro model of breast cancer. Taken together, these data support both direct and indirect roles for NE in promoting tumor cell proliferation.
In addition to proliferation, $\mathrm{NE}$ has been shown to enhance the migratory and invasive abilities of malignant cells $[69,72]$. In the study by Gaida et al. [72], the authors demonstrated that co-culture of pancreatic ductal adenocarcinoma (PDAC) cells with PMN resulted in dyshesion of malignant cells within the cultured monolayer. This phenotype was replicated by exogenous administration of NE $(3 \mu \mathrm{g} / \mathrm{ml})$ [72]. This dyshesion was associated with increased migration in vitro, which was attributed to loss of cellular expression of E-cadherin [72]. This enhanced migratory phenotype was similarly attenuated by exogenous inhibition of NE [72]. The invasive potential of PDAC cells, demonstrated by using a Matrigel trans-well assay, was similarly increased by the addition of NE $(1 \mu \mathrm{g} /$ $\mathrm{ml})$. This was associated with reduced cell surface expression of E-cadherin as with the migration assays mentioned above [72].

The migratory activity of esophageal cancer cells has similarly been shown to be increased by NE [73]. Wada et al. demonstrated a $20 \%$ increase in migration of TC-13 cells after exogenous administration of $5 \mu \mathrm{g} / \mathrm{ml}$ of NE. As with proliferation, this was abrogated by exogenous inhibition of NE with sivelestat [73].

In light of these in vitro findings, it is not surprising that $\mathrm{NE}$ has also been implicated in tumor progression in vivo [76]. Houghton et al. [76] demonstrated reduced tumor growth in an orthotopic model of lung cancer in NE-/mice compared to WT. The authors measured tumor burden within murine lungs 28 weeks following adenoviral infection of K-ras conditional knockout mice [76]. In NE $-/-$ mice, an approximate $30 \%$ reduction in pulmonary tumor area was observed compared to NE WT mice. The increase in pulmonary area covered by tumor in NE WT mice was associated with an increased rate of neoplastic cell proliferation as evidenced by increased Ki-67 staining compared to $\mathrm{NE}-/-$ mice [76]. As with the in vitro experiments outlined above, this was associated with enhanced signaling via PI3K in NE WT compared to NE -/- mice [76].

Kaplan-Meier survival analysis revealed that while none of the NE $-/-$ mice died over the study period, nearly all of the NE WT mice did. Thus, not only did NE appear to promote tumor growth, but it was also associated with a significantly more lethal phenotype [76].

\section{Neutrophil extracellular traps and metastatic progression}

Current studies specifically addressing the contribution of NETs to metastasis remain scarce. However, a large body of literature describing elevated levels of plasma cell-free DNA (cfDNA) in patients with breast, lung, stomach and 
colon cancers has been published [78-85]. However, the source of this cfDNA remains incompletely elucidated, and while some of it is tumor derived, there is evidence to suggest that neutrophil-derived DNA may also contribute. From a clinical standpoint, cfDNA monitoring may prove useful in monitoring patient response to therapy, tumor recurrence and prognostication [78-85]. This association with tumor progression is particularly fascinating given the recently described contribution of NETs. Accordingly, despite the fact that no studies unequivocally highlight these circulating nucleic acids as NETs, the data linking cfDNA concentration and prognosis are worth highlighting.

Nucleosomes represent one form of cfDNA comprising double-stranded DNA (dsDNA) and histone [78-85]. They can originate from multiple sources including the primary tumor as well as stromal cells and leukocytes [85]. As with circulating DNA, nucleosomes are elevated in cancer patients compared to healthy controls [78-85]. In addition, quantitative analysis of this nuclear material reveals that it may be of prognostic significance, predicting progressionfree and overall survival [78-85]. However, the research to date has fixated on tumor cells as the predominant source of circulating DNA. While this may contribute significantly to overall cfDNA levels, leukocyte-derived sources have also been demonstrated [80].

In the study by Fuchs et al. [80], the authors quantified plasma nucleosomes in patients with thrombotic microangiopathy (TMA) related to a variety of conditions including malignancy. TMAs are known complications of advanced malignancy and the authors postulate that neutrophilderived nucleosomes (e.g., NETs) contribute to this pathological state [80]. Accordingly, TMA patients were found to have significantly increased levels of plasma nucleosomes compared to healthy controls ( $\sim 10$ to 100-fold increase) [80]. In particular, seven of the eight TMA patients with underlying malignancy as the suspect root cause were found to have elevated levels of plasma nucleosomes ( $\sim 100$-fold versus control) [80]. In keeping with the hypothesis that plasma nucleosomes are at least in part neutrophil derived, the authors demonstrated elevated levels of plasma MPO and S100A8/A9 (calprotectin) in TMA patients compared to healthy controls [80]. Again, as with nucleosomes, patients with tumor-associated TMA exhibited significantly higher levels of these neutrophil markers than did healthy controls [80].

The observation that cfDNA can be neutrophil derived and consistent with NETs implies that other factors are at play in addition to passive release from developing tumors [80]. In light of what has been shown with regard to neutrophils, NETs and the tumor microenvironment, cfDNA may represent a host-tumor response with profound potential implications on tumor progression $[32,80]$.
In keeping with a possible pro-neoplastic role for NETs in tumor progression, multiple explanations can be put forward. First, NET components such as MMP-9, CG and NE may act at the level of distant metastases in an analogous manner to what has been described at the level of the primary tumor $[38-42,60,70,73,86]$. Furthermore, NETs are recognized by a number of leukocytes including macrophages and dendritic cells [12, 23, 87, 88]. Following their stimulation with NETs, macrophages can adopt proor anti-inflammatory cytokine expression profiles. These seemingly contradictory effects have led many authors to postulate that inappropriate or excessive NET deposition is associated with ongoing inflammation and tissue damage as opposed to its resolution [12, 17, 87, 88]. This persistent inflammatory state may result in ongoing expression of adhesion molecules facilitating tumor cell entrapment and interaction with the ECM [89-92]. In addition, areas of dense NET deposition could be rich in pro-tumorigenic proteins as previously described. In this manner, excessive NET deposition could create a "pre-metastatic niche". Concurrently, NETs themselves may play an adhesive role in an analogous manner to what has been described in microbial trapping. This physical interaction in a microenvironment rich in pro-tumorigenic proteins could similarly favor an aggressive neoplastic phenotype [32].

Neutrophil extracellular traps and adhesion

Adhesive events between disseminated tumor cells and end organ vasculature have been shown to play an important role in supporting the development of gross metastasis. Multiple studies including a number from our own research group, have revealed that neutrophils play a central role in circulating tumor cell (CTC) arrest. This has been demonstrated in in vitro models under conditions of flow as well as in vivo where they have been shown to facilitate CTC adhesion to both pulmonary and hepatic endothelial surfaces [22, 32, 90, 92, 93]. Under infectious/inflammatory conditions; not unlike those that support NET elaboration from neutrophils, such interactions appear to be enhanced $[90,92,94]$. Given that one of the primary roles attributed to NETs is the sequestration of intravascular bacteria, the possibility that NETs may act to capture CTCs in an analogous manner logically arises.

Our group tested this hypothesis directly both in vitro and in vivo. Under static conditions, tumor cell adhesion to neutrophil monolayers was enhanced four to fivefold following neutrophil pretreatment with PMA to induce NET formation compared to untreated controls. For example, following intrasplenic injection of LLC cells in septic mice, enhanced tumor cell arrest within the hepatic microvasculature can be directly visualized in vivo compared to healthy controls [90, 92]. Furthermore, CTC arrest 
A

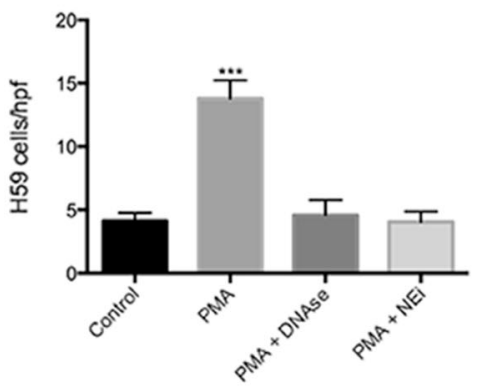

B

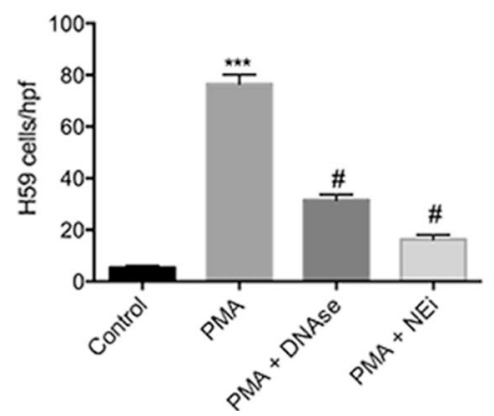

Fig. 1 NETs trap both human and murine tumor cells in vitro. a Under static or b dynamic conditions $\left(1\right.$ dyne/cm s$\left.{ }^{-1}\right)$, H59 and A549 cells demonstrate increased adhesion to NETs compared to unstimulated neutrophils. Addition of DNAse 1 (1000 U) or pretreatment of neutrophils with NEi results in levels of adhesion comparable to the control. Data are presented as mean \pm SEM. $* * * p<0.01$ versus control, DNAse and NEi. ${ }^{\#} p<0.05$ versus control. $\mathbf{c}$ Confocal imaging reveals that neoplastic cells (A549 red) become trapped

frequently occurs after direct contact with intravascular neutrophils, whose recruitment is enhanced during systemic infection. Depletion of neutrophils abrogates this effect and results in diminished overall CTC adhesion within the liver [90, 92]. In addition, the development of gross metastasis is decreased suggesting that increased adhesive events via direct CTC-neutrophil contact are an important precursor to the development of metastatic disease $[90,92]$.

A recent study from our group suggests that in the context of systemic sepsis, NET deposition within the microvasculature of a host organ such as the lung or liver may promote adhesion of CTC. In vitro, PMA treatment of human neutrophils was used to induce NET formation. In the presence of NETs, adhesion of murine (H59) and human (A549) lung carcinoma cells was increased approximately four to fivefold compared to the control. Degradation of NETs using DNAse 1 or inhibition of NET formation by pretreatment of neutrophil monolayers with

\section{Neutrophil NETs A549}

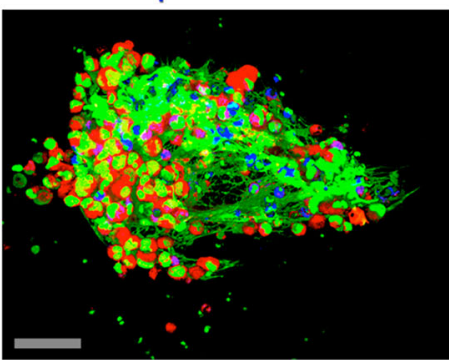

D

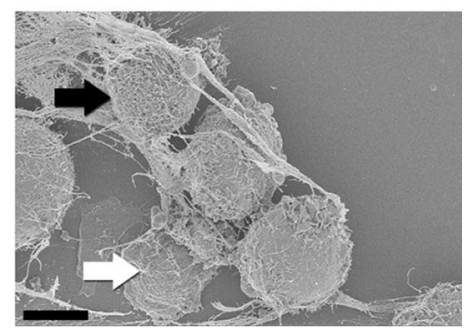

E

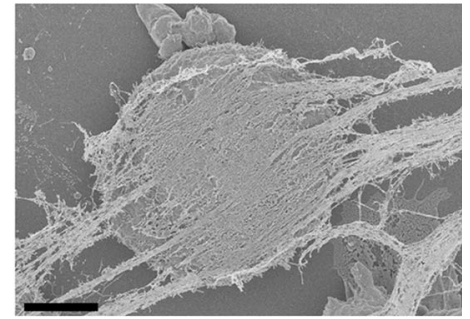

within webs of extracellular DNA (green) in proximity to neutrophils (blue). d-e Following co-incubation of A549- and PMA-stimulated neutrophils (white arrow), strands of extracellular DNA consistent with NETs (black arrow) can be seen in contact with neoplastic cells. At a higher magnification e, NETs are visualized in direct contact with adherent A549 tumor cells. Scale bars represent $40 \mu \mathrm{m}$ for confocal microscopy; $5 \mu \mathrm{m}$ for electron microscopy. The figure was reproduced with permission from the JCI [32]

NEi abrogated the increased tumor adhesion observed after PMA stimulation in both cell lines [32]. Imaging with both confocal and electron microscopy in this study demonstrated cancer cells trapped within webs of extracellular DNA. These NETs appeared to be wrapped around adherent tumor cells and in direct contact with the tumor cell membrane. Neutrophils, however, appeared to be in proximity to, but not in direct contact with, the tumor cells, suggesting that an adhesive mechanism was mediated by trapping within NETs (Fig. 1).

To more closely approximate physiologic conditions and determine if tumor cell adhesion to NETs is maintained under conditions of flow, murine (H59) or human (A549) lung cancer cells were then perfused over neutrophil monolayers in a parallel-plate flow chamber at a rate of 1 dyne $/ \mathrm{cm} \mathrm{s}^{-1}$. Addition of PMA to the tumor cell perfusate resulted in rapid formation of NETs resulting in a significant increase in tumor cell adhesion compared to control (unpublished data). Adhesion was significantly 
A
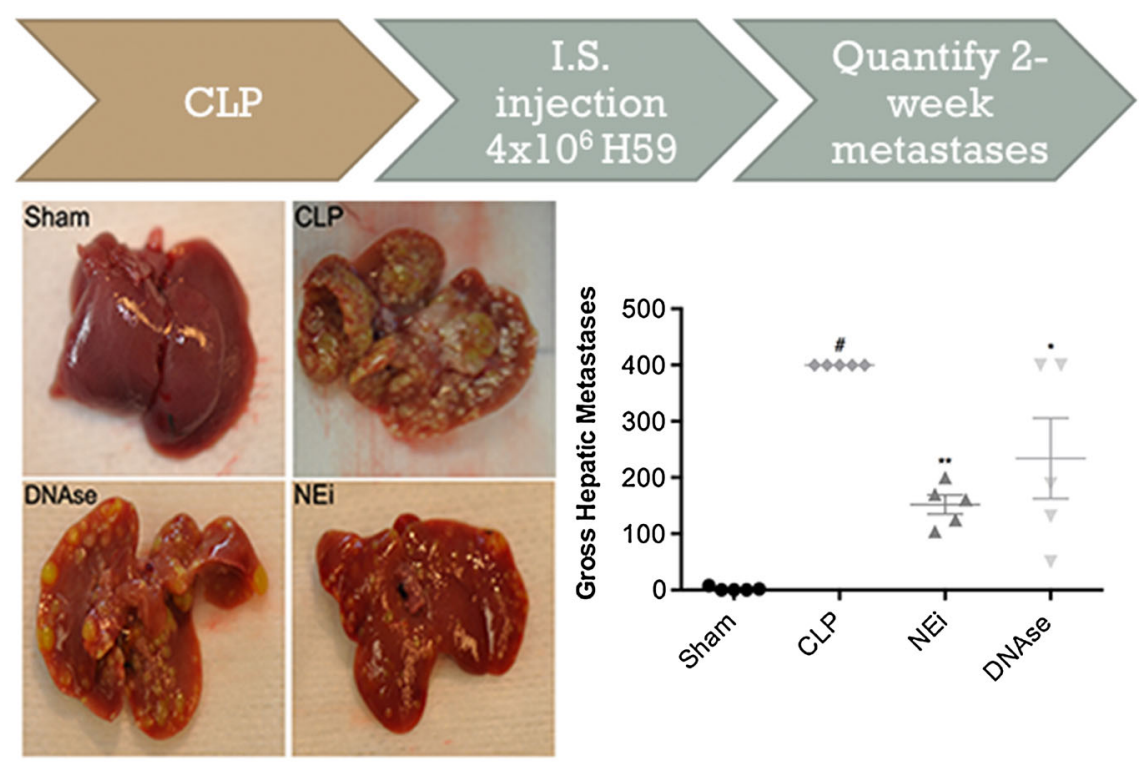

B
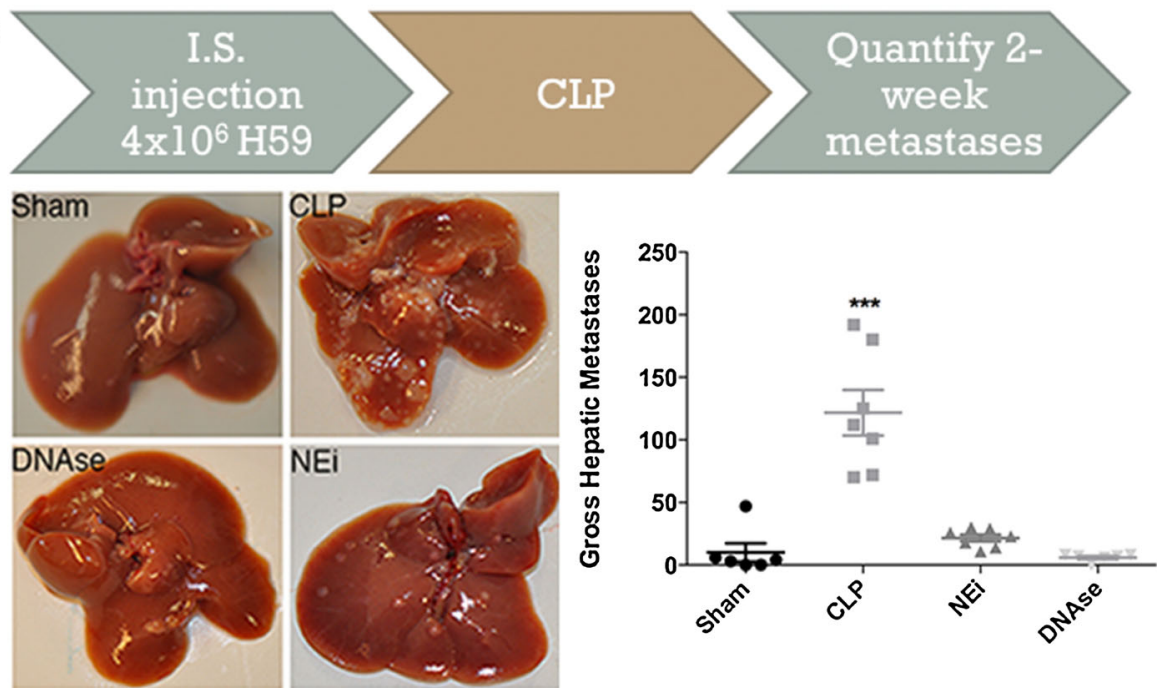

Fig. 2 Systemic sepsis promotes the development of gross metastasis, which is attenuated by systemic administration of inhibitors of NET formation. a Mice were subject to CLP to induce sepsis. Intrasplenic injection of H59 LLC cells was performed $24 \mathrm{~h}$ later. Administration of DNAse 1 intramuscular or NEi per os was started $24 \mathrm{~h}$ prior to CLP and continued daily for 14 days. At 14 days, mice were killed and the number of gross hepatic metastases was quantified. Representative images of hepatic nodules after necropsy in mice subject to sham surgery, CLP, CLP with daily DNAse 1 administration and CLP with daily NEi administration are shown. Data are presented as mean \pm SEM from the $n=5$ mice/group. ${ }^{*} p<0.05, * * p<0.01$ versus CLP. ${ }^{\#} p<0.001$ versus sham as determined by one way ANOVA with Tukey's HSD post hoc analysis. b To eliminate the contribution of early adhesive events,

mice received intrasplenic injection of $\mathrm{H} 59$ cells $24 \mathrm{~h}$ prior to CLP. $24 \mathrm{~h}$ later, CLP or sham surgery was performed. NEi and DNAse treatment began the day prior to surgery and continued for 14 days as in (a). Necropsy at 2 weeks permitted quantification of gross hepatic metastasis. Representative images are shown. In the experiments outlined in both (a) and (b), CLP resulted in a significant increase in the number of gross metastatic nodules compared to sham. Treatment with DNAse or NEi after CLP resulted in a significant decrease in the number of gross metastases. Data are presented as mean \pm SEM from $n=6-7$ mice/group. $* * * p<0.001$ versus sham, NEi and DNAse as determined by one-way ANOVA with Tukey's HSD post hoc analysis. The figure was adapted and reproduced with permission from the JCI [32]

reduced by addition of DNAse 1 to the perfusate or by pretreatment of neutrophils with NEi.

Similar observations were made in vivo following treatment of mice with cecal ligation and puncture (CLP) to induce systemic sepsis and stimulate widespread NET

deposition. Following injection of murine lung (H59) or melanoma (B16-F10) cells via the tail vein, tumor-NET interactions were quantified and visualized using spinning disc confocal intravital microscopy (SD-IVM). Tumor arrest was significantly increased after CLP 
compared to mice subject to sham surgery or those administered DNAse 1 or NEi after CLP. Furthermore, direct tumor-NET interactions could be visualized suggesting a mechanical trapping effect of NETs on tumor cells following their interaction. In response to systemic infection, widespread NET deposition occurs within end organ microvasculature such as the hepatic sinusoidal spaces and pulmonary capillaries. NET deposition is vulnerable to pharmacologic inhibition with DNAse and NEi. Furthermore, intravascular NET deposition is abolished by neutrophil depletion. If septic mice are subject to IV administration of LLC cells, gross hepatic metastasis formation is significantly enhanced compared to non-septic mice (Fig. 2). However, following administration of DNAse or NEi to septic mice, in an attempt to diminish NET deposition, gross hepatic metastasis formation was attenuated. These findings mirror what was observed with respect to NET deposition with end organ microvasculature suggesting that NETs may play a role in trapping CTC.

The effect of NET deposition on metastasis goes beyond early adhesive events

Following dissemination and adhesion, CTC must be able to proliferate to form stable metastatic foci. The study by Sangaletti et al. [34] suggests that NETs may play a direct proliferative role and in fact may inhibit apoptosis under certain conditions. In this manner, they may act to promote metastasis formation. Data from our own group support this finding. In a previous study, we demonstrate that following sequestration within NETs, CTCs are able to form stable micro metastatic foci and, as previously stated, ultimately go on to form macro metastases. This implies that trapped cells are able not only to survive interactions with NETs, but are also able to grow. Furthermore, if mice were injected with LLC cells prior to the septic insult, hepatic metastatic disease burden was still abrogated by DNAse and NEi administration. In this model, the contribution of early adhesive events to metastasis formation was eliminated (Fig. 2). At the very least, this result suggests that NETs can influence tumor progression in multiple ways. Taken together, these data support a pro-metastatic role for NETs; however the mechanisms by which NETs may act to produce clinically apparent metastasis are multiple. First, they may act simply by promoting early adhesive events, thus increasing sequestration of malignant cells in end organs leading to a greater number of metastases. Additionally, NETs may act by directly promoting a more malignant phenotype in neoplastic cells as a consequence of their interaction.

\section{Neutrophil extracellular traps as therapeutic targets}

In keeping with the hypothesis that NETs provide a microenvironment favoring interactions leading to tumor cell dissemination and metastasis, it stands to reason that disrupting NETs may hinder these processes. Our group has shown that disruption of NETs using either DNAse or NEi abrogates adhesion and metastasis formation highlighting NETs as a possible therapeutic target.

DNAse has been used in human disease for the treatment of such conditions as empyema and cystic fibrosis following administration via the intra-pleural or inhaled routes, respectively. In addition to demonstrating safety, the clinical results have been encouraging to date. Similarly, its systemic administration in the context of systemic lupus erythematosus (SLE) has been attempted without adverse effects [95]. These studies serve to highlight its potential safety for use in human cancer trials. However, to the best of our knowledge, no such studies have been performed to date. Conversely, a number of animal studies have been performed showing an anti-metastatic effect following the administration of DNAse 1 in a variety of tumor models. Sugihara et al. demonstrated reduced hepatic metastasis formation in a murine model of lymphoma following DNAse 1 administration. In particular, the authors noted that DNAse 1 administration was associated with reduced intravascular arrest of neoplastic cells compared to untreated control mice [96]. Patutina et al. demonstrate similar findings in a murine model of LLCinduced pulmonary metastases. Herein, the authors demonstrate a greater than threefold reduction in the number of surface metastasis in tumor-bearing mice administered DNAse compared to untreated controls [97].

With regard to NEi, no studies have examined its antineoplastic effects in humans. However, excessive NE function, which is required for NET elaboration, may be associated with tumor development and progression in human cancers [74]. A recent Lancet Oncology review highlights the frequency of $\alpha 1$-antitrypsin deficiency heterozygotes in a variety of cancers including colon, lung and bladder tumors [74]. The authors postulate that because such individuals have diminished levels of this negative regulator of $\mathrm{NE}$, its unfettered activity may stimulate tumor development and progression [74]. Accordingly, elevated levels of NE have been demonstrated in a variety of cancers including lung and breast $[74,86,98]$. The levels have been shown to correlate with both tumor stage and progression-free survival [74, 86, 98]. With respect to bronchoalveolar adenocarcinoma (BAC), elevated levels of $\mathrm{NE}$ have been shown to enhance tumor cell shedding and thus possibly intrapulmonary spread [86]. Accordingly, cumulative survival in 
BAC patients has been shown to correlate with tumor shedding and thus potentially NE [86].

Elevated serum levels of NE have been demonstrated following both pulmonary and esophageal surgical procedures [99-102]. This elevation may be associated with adverse outcomes relating to pulmonary function following thoracic surgical procedures such as acute respiratory distress syndrome (ARDS) [99-102]. Accordingly, a number of studies have been performed examining both the safety of perioperative sivelestat and its efficacy in preventing postoperative adverse events [99-102]. Kawahara et al. [102] performed a randomized controlled trial (RCT) in which 22 patients were randomized to receive perioperative sivelestat $(n=11)$ or placebo $(n=11)$ for video-assisted thoracic surgery (VATS) for primary lung cancer. The authors demonstrated that perioperative sivelestat was safe and associated with a significantly reduced duration of systemic inflammatory response syndrome (SIRS) compared to placebo as determined by heart rate and $\mathrm{P} / \mathrm{F}$ ratio [102]. In addition, postoperative IL-8 levels were significantly reduced in sivelestat-treated patients compared to those who received placebo and IL-8 is a known inducer of NETs [102].

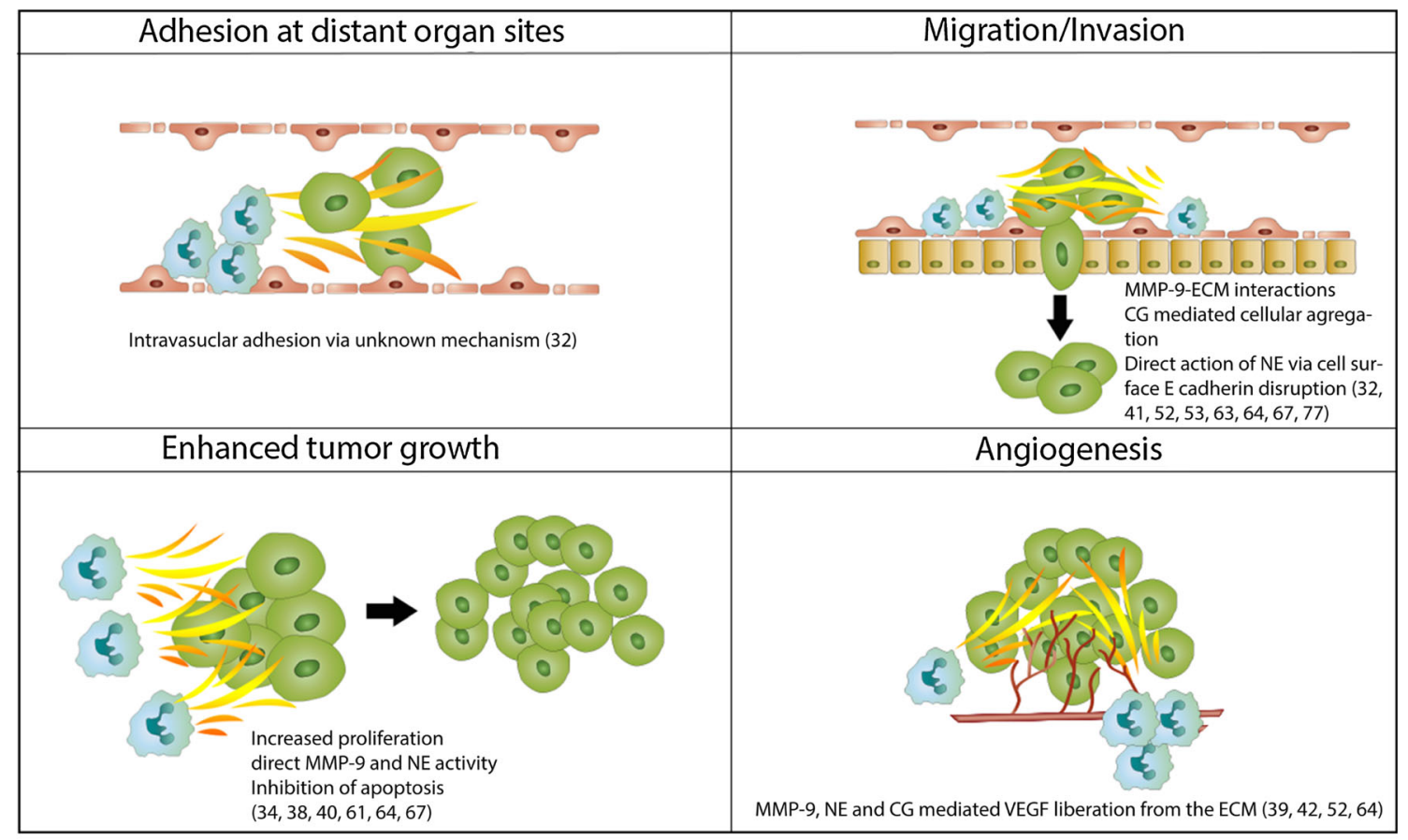

Legend

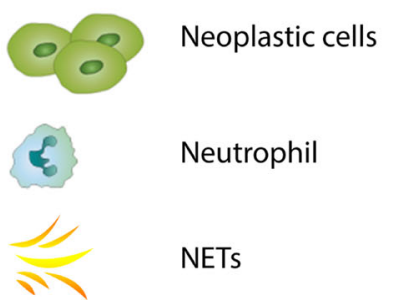

Fig. 3 Postulated pro-tumorigenic roles of NETs. NETs are composed of extruded neutrophil-derived chromatin decorated in antimicrobial proteins and peptides. These include MMP-9, CG and $\mathrm{NE}$, all of which have demonstrated roles in multiple stages of the metastatic cascade. It is possible that NETs provide a structure on which these peptides are able to interact with neoplastic cells. In this manner, NETs may provide a microenvironment with a high local concentration of pro-tumorigenic proteins, which favor their interaction with tumor cells by bringing them into close proximity with one another. Possible pro-neoplastic effector mechanisms include intravascular adhesion, enhanced tumor cell growth, enhanced migration and invasion, and increased angiogenesis. Direct intravascular adhesion has been demonstrated between NETs and neoplastic cells, both in vitro and in vivo [32]. Enhanced tumor growth may occur via increased proliferation directly. This could possibly be mediated by the direct activity of MMP-9 and NE. In addition, anti-apoptotic effects of NETs on neoplastic cells have been demonstrated [34, 38, $40,70,73,76]$. NETs could act to facilitate migration and invasion, thus promoting the development of metastases. Possible mechanisms include MMP-9-ECM interactions, CG-mediated cellular aggregate formation and E-cadherin disruption via $\mathrm{NE}[32,41,61,62,72,73$, $76,86]$. Finally, angiogenesis could be promoted via MMP-9 and NEand CG-mediated VEGF liberation from the ECM [34, 38, 40, 70, 73, 76] 
The RCT by Makino et al. [100] randomized 31 patients undergoing VATS esophagectomy for malignant disease to receive perioperative sivelestat $(n=16)$ or saline $(n=15)$. Postoperatively, patients in the sivelestat group experienced reduced duration of mechanical ventilation, reduced duration of SIRS, reduced length of ICU stay and a trend toward reduced overall hospital stay [100]. Furthermore, patients in the sivelestat group demonstrated a more rapid reduction in postoperative $\mathrm{C}$-reactive protein (CRP) [100]. Taken together, the results of these studies suggest that perioperative $\mathrm{NE}$ inhibition may act to reduce inflammatory burden on patients resulting in improved clinical outcomes. In keeping with the hypothesis that the systemic inflammatory insult that follows surgery may act to promote tumor progression, it would be interesting to know if this is also associated with tumor progression. However, no such studies have been performed to date.

\section{Conclusions}

Neutrophils have long been recognized for their important role in host defense $[8,103]$, and as outlined above these biologically and functionally diverse cells profoundly influence the course of infectious processes. Recent evidence has re-introduced the neutrophil as a potential key player in influencing a number of neoplastic processes in addition to their role in host defense from infection. With regard to cancer, seemingly opposite roles have been demonstrated [104]. However, the scope of neutrophil responses appears to be broader than previously thought and the possibility that neutrophils can exhibit diverse phenotypes is beginning to take hold. This being said, some evidence strongly suggests that neutrophils can take on behaviors in the context of tumor progression that are catastrophic to the host.

Unfortunately, no clinical interventions are currently targeted toward neutrophil effector function despite recent data suggesting that neutrophil responses may favor tumor progression. However, as our understanding of protumorigenic neutrophil effector mechanisms improves, the possibility of mitigating their deleterious impact on patients' oncologic outcome arises.

The mechanisms underlying the apparent pro-metastatic role for NETs may be multiple. As highlighted above, factors elaborated by neutrophils can support tumor development at every step of the metastatic cascade, from early adhesion, proliferation and invasion to angiogenesis. Many of these factors have been demonstrated within NETs. Accordingly, by sequestering tumor cells and bringing them into close proximity with a number of neutrophil-derived factors, NETs may generate a microenvironment for the trapped tumor cell rich in proteins and enzymes that facilitate their progression. In this way, NETs may represent a common mechanism by which a variety of neutrophil-derived factors exert their function on tumor biology (Fig. 3).

In keeping with this conceptualization, drugs that degrade NETs (DNAse) or NEi demonstrate a profound inhibitory effect on the development of metastatic disease in the models employed. This particular observation is of potential clinical significance, as both classes of drugs have been tested in humans and have demonstrated safety and efficacy in the treatment of cystic fibrosis (DNAse), empyema (DNAse) and the inhibition of post-esophagectomy ARDS (sivelestat) $(6868,[100,105,106])$. This not only introduces NETs as a potential therapeutic target, but also offers a potential therapeutic avenue to combat their negative effects.

\section{References}

1. Colotta F, Allavena P, Sica A, Garlanda C, Mantovani A (2009) Cancer-related inflammation, the seventh hallmark of cancer: links to genetic instability. Carcinogenesis 30:1073-1081

2. Smith HA, Kang Y (2013) The metastasis-promoting roles of tumor-associated immune cells. J Mol Med (Berl) 91:411-429

3. Fridlender ZG, Sun J, Kim S, Kapoor V, Cheng G, Ling L, Worthen GS, Albelda SM (2009) Polarization of tumor-associated neutrophil phenotype by TGF-beta: "N1" versus "N2" TAN. Cancer Cell 16:183-194

4. Kusmartsev S, Nagaraj S, Gabrilovich DI (2005) Tumor-associated CD8 $+\mathrm{T}$ cell tolerance induced by bone marrow-derived immature myeloid cells. J Immunol 175:4583-4592

5. Ostrand-Rosenberg S, Sinha P (2009) Myeloid-derived suppressor cells: linking inflammation and cancer. J Immunol 182:4499-4506

6. Fridlender ZG, Albelda SM (2012) Tumor associated neutrophils: friend or foe? Carcinogenesis 33:949-955

7. Piccard H, Muschel RJ, Opdenakker G (2012) On the dual roles and polarized phenotypes of neutrophils in tumor development and progression. Crit Rev Oncol Hematol 82:296-309

8. Cavaillon JM (2011) The historical milestones in the understanding of leukocyte biology initiated by Elie Metchnikoff. J Leukoc Biol 90:413-424

9. Fridlender ZG, Sun J, Mishalian I, Singhal S, Cheng G, Kapoor V, Horng W, Fridlender G, Bayuh R, Worthen GS et al (2012) Transcriptomic analysis comparing tumor-associated neutrophils with granulocytic myeloid-derived suppressor cells and normal neutrophils. PLoS One 7:e31524

10. Urban CF, Ermert D, Schmid M, Abu-Abed U, Goosmann C, Nacken W, Brinkmann V, Jungblut PR, Zychlinsky A (2009) Neutrophil extracellular traps contain calprotectin, a cytosolic protein complex involved in host defense against Candida albicans. PLoS Pathog 5:e1000639

11. Saitoh T, Komano J, Saitoh Y, Misawa T, Takahama M, Kozaki T, Uehata T, Iwasaki H, Omori H, Yamaoka S et al (2012) Neutrophil extracellular traps mediate a host defense response to human immunodeficiency virus-1. Cell Host Microbe 12:109-116

12. Papayannopoulos V, Zychlinsky A (2009) NETs: a new strategy for using old weapons. Trends Immunol 30:513-521 
13. Fuchs TA, Abed U, Goosmann C, Hurwitz R, Schulze I, Wahn V, Weinrauch Y, Brinkmann V, Zychlinsky A (2007) Novel cell death program leads to neutrophil extracellular traps. J Cell Biol 176:231-241

14. Brinkmann V, Zychlinsky A (2012) Neutrophil extracellular traps: is immunity the second function of chromatin? J Cell Biol 198:773-783

15. Brinkmann V, Reichard U, Goosmann C, Fauler B, Uhlemann Y, Weiss DS, Weinrauch Y, Zychlinsky A (2004) Neutrophil extracellular traps kill bacteria. Science 303:1532-1535

16. Kolaczkowska E, Kubes P (2013) Neutrophil recruitment and function in health and inflammation. Nat Rev Immunol 13:159-175

17. McDonald B, Urrutia R, Yipp BG, Jenne CN, Kubes P (2012) Intravascular Neutrophil Extracellular Traps Capture Bacteria from the Bloodstream during Sepsis. Cell Host Microbe 12:324-333

18. Pilsczek FH, Salina D, Poon KK, Fahey C, Yipp BG, Sibley CD, Robbins SM, Green FH, Surette MG, Sugai M et al (2010) A novel mechanism of rapid nuclear neutrophil extracellular trap formation in response to Staphylococcus aureus. J Immunol 185:7413-7425

19. Clark SR, Ma AC, Tavener SA, McDonald B, Goodarzi Z, Kelly MM, Patel KD, Chakrabarti S, McAvoy E, Sinclair GD et al (2007) Platelet TLR4 activates neutrophil extracellular traps to ensnare bacteria in septic blood. Nat Med 13:463-469

20. Mantovani A, Cassatella MA, Costantini C, Jaillon S (2011) Neutrophils in the activation and regulation of innate and adaptive immunity. Nat Rev Immunol 11:519-531

21. Colotta F, Re F, Polentarutti N, Sozzani S, Mantovani A (1992) Modulation of granulocyte survival and programmed cell death by cytokines and bacterial products. Blood 80:2012-2020

22. Huh SJ, Liang S, Sharma A, Dong C, Robertson GP (2010) Transiently entrapped circulating tumor cells interact with neutrophils to facilitate lung metastasis development. Cancer Res 70:6071-6082

23. Phillipson M, Kubes P (2011) The neutrophil in vascular inflammation. Nat Med 17:1381-1390

24. Brinkmann V, Laube B, Abu Abed U, Goosmann C, Zychlinsky A (2010) Neutrophil extracellular traps: how to generate and visualize them. J Vis Exp 36. doi:10.3791/1724

25. Neeli I, Dwivedi N, Khan S, Radic M (2009) Regulation of extracellular chromatin release from neutrophils. J Innate Immun 1:194-201

26. Li Y, Liu B, Fukudome EY, Lu J, Chong W, Jin G, Liu Z, Velmahos GC, Demoya M, King DR et al (2011) Identification of citrullinated histone $\mathrm{H} 3$ as a potential serum protein biomarker in a lethal model of lipopolysaccharide-induced shock. Surgery 150:442-451

27. Munafo DB, Johnson JL, Brzezinska AA, Ellis BA, Wood MR, Catz SD (2009) DNase I inhibits a late phase of reactive oxygen species production in neutrophils. J Innate Immun 1:527-542

28. Hakkim A, Fuchs TA, Martinez NE, Hess S, Prinz H, Zychlinsky A, Waldmann H (2011) Activation of the Raf-MEK-ERK pathway is required for neutrophil extracellular trap formation. Nat Chem Biol 7:75-77

29. Papayannopoulos V, Metzler KD, Hakkim A, Zychlinsky A (2010) Neutrophil elastase and myeloperoxidase regulate the formation of neutrophil extracellular traps. J Cell Biol 191:677-691

30. Metzler KD, Fuchs TA, Nauseef WM, Reumaux D, Roesler J, Schulze I, Wahn V, Papayannopoulos V, Zychlinsky A (2011) Myeloperoxidase is required for neutrophil extracellular trap formation: implications for innate immunity. Blood 117:953-959
31. Pham CT (2006) Neutrophil serine proteases: specific regulators of inflammation. Nat Rev Immunol 6:541-550

32. Cools-Lartigue J, Spicer J, McDonald B, Gowing S, Chow S, Giannias B, Bourdeau F, Kubes P, Ferri L (2013) Neutrophil extracellular traps sequester circulating tumor cells and promote metastasis. J Clin Invest 123:3446-3458

33. Berger-Achituv S, Brinkmann V, Abed UA, Kuhn LI, Ben-Ezra J, Elhasid R, Zychlinsky A (2013) A proposed role for neutrophil extracellular traps in cancer immunoediting. Front Immunol 4:48

34. Sangaletti S, Tripodo C, Vitali C, Portararo P, Guarnotta C, Casalini P, Cappetti B, Miotti S, Pinciroli P, Fuligni F et al (2014) Defective stromal remodeling and neutrophil extracellular traps in lymphoid tissues favor the transition from autoimmunity to lymphoma. Cancer Discov 4:110-129

35. Ho-Tin-Noe B, Carbo C, Demers M, Cifuni SM, Goerge T, Wagner DD (2009) Innate immune cells induce hemorrhage in tumors during thrombocytopenia. Am J Pathol 175:1699-1708

36. Demers M, Krause DS, Schatzberg D, Martinod K, Voorhees JR, Fuchs TA, Scadden DT, Wagner DD (2012) Cancers predispose neutrophils to release extracellular DNA traps that contribute to cancer-associated thrombosis. Proc Natl Acad Sci USA 109:13076-13081

37. Mishalian I, Bayuh R, Levy L, Zolotarov L, Michaeli J, Fridlender ZG (2013) Tumor-associated neutrophils (TAN) develop pro-tumorigenic properties during tumor progression. Cancer Immunol Immunother 62:1745-1756

38. Acuff HB, Carter KJ, Fingleton B, Gorden DL, Matrisian LM (2006) Matrix metalloproteinase-9 from bone marrow-derived cells contributes to survival but not growth of tumor cells in the lung microenvironment. Cancer Res 66:259-266

39. Bergers G, Brekken R, McMahon G, Vu TH, Itoh T, Tamaki K, Tanzawa K, Thorpe P, Itohara S, Werb Z et al (2000) Matrix metalloproteinase- 9 triggers the angiogenic switch during carcinogenesis. Nat Cell Biol 2:737-744

40. Coussens LM, Tinkle CL, Hanahan D, Werb Z (2000) MMP-9 supplied by bone marrow-derived cells contributes to skin carcinogenesis. Cell 103:481-490

41. Masson V, de la Ballina LR, Munaut C, Wielockx B, Jost M, Maillard C, Blacher S, Bajou K, Itoh T, Itohara S et al (2005) Contribution of host MMP-2 and MMP-9 to promote tumor vascularization and invasion of malignant keratinocytes. FASEB J 19:234-236

42. Nozawa H, Chiu C, Hanahan D (2006) Infiltrating neutrophils mediate the initial angiogenic switch in a mouse model of multistage carcinogenesis. Proc Natl Acad Sci USA 103:12493-12498

43. Van Coillie E, Van Aelst I, Wuyts A, Vercauteren R, Devos R, De Wolf-Peeters C, Van Damme J, Opdenakker G (2001) Tumor angiogenesis induced by granulocyte chemotactic protein-2 as a countercurrent principle. Am J Pathol 159:1405-1414

44. Daley JM, Thomay AA, Connolly MD, Reichner JS, Albina JE (2008) Use of Ly6G-specific monoclonal antibody to deplete neutrophils in mice. J Leukoc Biol 83(1):64-70

45. Melnikov VY, Faubel S, Siegmund B, Lucia MS, Ljubanovic D, Edelstein CL (2002) Neutrophil-independent mechanisms of caspase-1 and IL-18-mediated ischemic acute tubular necrosis in mice. J Clin Invest 110:1083-1091

46. Zhou J, Stohlman SA, Hinton DR, Marten NW (2003) Neutrophils promote mononuclear cell infiltration during viral-induced encephalitis. J Immunol 170:3331-3336

47. Mednick AJ, Feldmesser M, Rivera J, Casadevall A (2003) Neutropenia alters lung cytokine production in mice and reduces their susceptibility to pulmonary cryptococcosis. Eur J Immunol 33:1744-1753 
48. Rivero-Nava L, Aguirre-Garcia J, Shibayama-Salas M, Hernandez-Pando R, Tsutsumi V, Calderon J (2002) Entamoeba histolytica: acute granulomatous intestinal lesions in normal and neutrophil-depleted mice. Exp Parasitol 101:183-192

49. Chen L, Watanabe T, Watanabe H, Sendo F (2001) Neutrophil depletion exacerbates experimental Chagas' disease in BALB/c, but protects C57BL/6 mice through modulating the Th1/Th2 dichotomy in different directions. Eur J Immunol 31:265-275

50. Tateda K, Moore TA, Deng JC, Newstead MW, Zeng X, Matsukawa A et al (2001) Early recruitment of neutrophils determines subsequent $\mathrm{T} 1 / \mathrm{T} 2$ host responses in a murine model of Legionella pneumophila pneumonia. J Immunol 166:3355-3361

51. Stirling DP, Liu S, Kubes P, Yong VW (2009) Depletion of Ly6G/Gr-1 leukocytes after spinal cord injury in mice alters wound healing and worsens neurological outcome. J Neurosci 29(3):753-764

52. Rose S, Misharin A, Perlman H (2012) A novel Ly6C/Ly6Gbased strategy to analyze the mouse splenic myeloid compartment. Cytom A 81(4):343-350

53. Borregaard N, Sorensen OE, Theilgaard-Monch K (2007) Neutrophil granules: a library of innate immunity proteins. Trends Immunol 28:340-345

54. Segal AW (2005) How neutrophils kill microbes. Annu Rev Immunol 23:197-223

55. Campanelli D, Detmers PA, Nathan CF, Gabay JE (1990) Azurocidin and a homologous serine protease from neutrophils. Differential antimicrobial and proteolytic properties. J Clin Invest 85:904-915

56. Gabay JE, Almeida RP (1993) Antibiotic peptides and serine protease homologs in human polymorphonuclear leukocytes: defensins and azurocidin. Curr Opin Immunol 5:97-102

57. Korkmaz B, Moreau T, Gauthier F (2008) Neutrophil elastase, proteinase 3 and cathepsin G: physicochemical properties, activity and physiopathological functions. Biochimie 90:227-242

58. Scocchi M, Skerlavaj B, Romeo D, Gennaro R (1992) Proteolytic cleavage by neutrophil elastase converts inactive storage proforms to antibacterial bactenecins. Eur $\mathrm{J}$ Biochem 209:589-595

59. Morimoto-Kamata R, Mizoguchi S, Ichisugi T, Yui S (2012) Cathepsin $\mathrm{G}$ induces cell aggregation of human breast cancer MCF-7 cells via a 2-step mechanism: catalytic site-independent binding to the cell surface and enzymatic activity-dependent induction of the cell aggregation. Mediators Inflamm 2012:456462

60. Yui S, Tomita K, Kudo T, Ando S, Yamazaki M (2005) Induction of multicellular 3-D spheroids of MCF-7 breast carcinoma cells by neutrophil-derived cathepsin $\mathrm{G}$ and elastase. Cancer Sci 96:560-570

61. Wilson TJ, Nannuru KC, Futakuchi M, Singh RK (2010) Cathepsin G-mediated enhanced TGF-beta signaling promotes angiogenesis via upregulation of VEGF and MCP-1. Cancer Lett 288:162-169

62. Wilson TJ, Nannuru KC, Futakuchi M, Sadanandam A, Singh RK (2008) Cathepsin G enhances mammary tumor-induced osteolysis by generating soluble receptor activator of nuclear factor-kappaB ligand. Cancer Res 68:5803-5811

63. Maksimowicz T, Chyczewska E, Chyczewski L, Niklinski J, Ostrowska H, Szyszko J, Furman M (1997) Activity and tissue localization of cathepsin $\mathrm{G}$ in non small cell lung cancer. Rocz Akad Med Bialymst 42(Suppl 1):199-216

64. Klein CA (2008) Cancer. The metastasis cascade. Science 321:1785-1787

65. Belaaouaj A, McCarthy R, Baumann M, Gao Z, Ley TJ, Abraham SN, Shapiro SD (1998) Mice lacking neutrophil elastase reveal impaired host defense against gram negative bacterial sepsis. Nat Med 4:615-618

66. Tkalcevic J, Novelli M, Phylactides M, Iredale JP, Segal AW, Roes J (2000) Impaired immunity and enhanced resistance to endotoxin in the absence of neutrophil elastase and cathepsin G. Immunity 12:201-210

67. Wiesner J, Vilcinskas A (2010) Antimicrobial peptides: the ancient arm of the human immune system. Virulence 1:440-464

68. Hager M, Cowland JB, Borregaard N (2010) Neutrophil granules in health and disease. J Intern Med 268:25-34

69. Grosse-Steffen T, Giese T, Giese N, Longerich T, Schirmacher P, Hansch GM, Gaida MM (2012) Epithelial-to-mesenchymal transition in pancreatic ductal adenocarcinoma and pancreatic tumor cell lines: the role of neutrophils and neutrophil-derived elastase. Clin Dev Immunol 2012:720768

70. Gregory AD, Hale P, Perlmutter DH, Houghton AM (2012) Clathrin pit-mediated endocytosis of neutrophil elastase and cathepsin G by cancer cells. J Biol Chem 287:35341-35350

71. Sato T, Takahashi S, Mizumoto T, Harao M, Akizuki M, Takasugi M, Fukutomi T, Yamashita J (2006) Neutrophil elastase and cancer. Surg Oncol 15:217-222

72. Gaida MM, Steffen TG, Gunther F, Tschaharganeh DF, Felix K, Bergmann F, Schirmacher P, Hansch GM (2012) Polymorphonuclear neutrophils promote dyshesion of tumor cells and elastase-mediated degradation of E-cadherin in pancreatic tumors. Eur J Immunol 42:3369-3380

73. Wada Y, Yoshida K, Tsutani Y, Shigematsu H, Oeda M, Sanada Y, Suzuki T, Mizuiri H, Hamai Y, Tanabe K et al (2007) Neutrophil elastase induces cell proliferation and migration by the release of TGF-alpha, PDGF and VEGF in esophageal cell lines. Oncol Rep 17:161-167

74. Sun Z, Yang P (2004) Role of imbalance between neutrophil elastase and alpha 1-antitrypsin in cancer development and progression. Lancet Oncol 5:182-190

75. Wada Y, Yoshida K, Hihara J, Konishi K, Tanabe K, Ukon K, Taomoto J, Suzuki T, Mizuiri H (2006) Sivelestat, a specific neutrophil elastase inhibitor, suppresses the growth of gastric carcinoma cells by preventing the release of transforming growth factor-alpha. Cancer Sci 97:1037-1043

76. Houghton AM, Rzymkiewicz DM, Ji H, Gregory AD, Egea EE, Metz HE, Stolz DB, Land SR, Marconcini LA, Kliment CR et al (2010) Neutrophil elastase-mediated degradation of IRS-1 accelerates lung tumor growth. Nat Med 16:219-223

77. Nawa M, Osada S, Morimitsu K, Nonaka K, Futamura M, Kawaguchi Y, Yoshida K (2012) Growth effect of neutrophil elastase on breast cancer: favorable action of sivelestat and application to anti-HER2 therapy. Anticancer Res 32:13-19

78. Frattini M, Gallino G, Signoroni S, Balestra D, Battaglia L, Sozzi G, Leo E, Pilotti S, Pierotti MA (2006) Quantitative analysis of plasma DNA in colorectal cancer patients: a novel prognostic tool. Ann N Y Acad Sci 1075:185-190

79. Frattini M, Gallino G, Signoroni S, Balestra D, Lusa L, Battaglia L, Sozzi G, Bertario L, Leo E, Pilotti S et al (2008) Quantitative and qualitative characterization of plasma DNA identifies primary and recurrent colorectal cancer. Cancer Lett 263:170-181

80. Fuchs TA, Kremer Hovinga JA, Schatzberg D, Wagner DD, Lammle B (2012) Circulating DNA and myeloperoxidase indicate disease activity in patients with thrombotic microangiopathies. Blood 120:1157-1164

81. Holdenrieder S, Stieber P, von Pawel J, Raith H, Nagel D, Feldmann K, Seidel D (2004) Circulating nucleosomes predict the response to chemotherapy in patients with advanced nonsmall cell lung cancer. Clin Cancer Res 10:5981-5987

82. Kremer A, Holdenrieder S, Stieber P, Wilkowski R, Nagel D, Seidel D (2006) Nucleosomes in colorectal cancer patients during radiochemotherapy. Tumour Biol 27:235-242 
83. Kumar S, Guleria R, Singh V, Bharti AC, Mohan A, Das BC (2010) Plasma nucleosome levels might predict response to therapy in patients with advanced non-small-cell lung cancer. Clin Lung Cancer 11:36-44

84. Kuroi K, Tanaka C, Toi M (1999) Plasma Nucleosome Levels in Node-Negative Breast Cancer Patients. Breast Cancer 6:361-364

85. Esposito A, Bardelli A, Criscitiello C, Colombo N, Gelao L, Fumagalli L, Minchella I, Locatelli M, Goldhirsch A, Curigliano G (2014) Monitoring tumor-derived cell-free DNA in patients with solid tumors: Clinical perspectives and research opportunities. Cancer Treat Rev 40:648-655

86. Wislez M, Antoine M, Rabbe N, Gounant V, Poulot V, Lavole A, Fleury-Feith J, Cadranel J (2007) Neutrophils promote aerogenous spread of lung adenocarcinoma with bronchioloalveolar carcinoma features. Clin Cancer Res 13:3518-3527

87. Meng W, Paunel-Gorgulu A, Flohe S, Hoffmann A, Witte I, Mackenzie C, Baldus SE, Windolf J, Logters TT (2012) Depletion of neutrophil extracellular traps in vivo results in hypersusceptibility to polymicrobial sepsis in mice. Crit Care 16:R137

88. Narasaraju T, Yang E, Samy RP, Ng HH, Poh WP, Liew AA, Phoon MC, van Rooijen N, Chow VT (2011) Excessive neutrophils and neutrophil extracellular traps contribute to acute lung injury of influenza pneumonitis. Am J Pathol 179:199-210

89. Hsu RY, Chan CH, Spicer JD, Rousseau MC, Giannias B, Rousseau S, Ferri LE (2011) LPS-induced TLR4 signaling in human colorectal cancer cells increases beta1 integrin-mediated cell adhesion and liver metastasis. Cancer Res 71:1989-1998

90. McDonald B, Spicer J, Giannais B, Fallavollita L, Brodt P, Ferri LE (2009) Systemic inflammation increases cancer cell adhesion to hepatic sinusoids by neutrophil mediated mechanisms. Int $\mathbf{J}$ Cancer 125:1298-1305

91. Rousseau MC, Hsu RY, Spicer JD, McDonald B, Chan CH, Perera RM, Giannias B, Chow SC, Rousseau S, Law S et al (2013) Lipopolysaccharide-induced toll-like receptor 4 signaling enhances the migratory ability of human esophageal cancer cells in a selectin-dependent manner. Surgery 154:69-77

92. Spicer JD, McDonald B, Cools-Lartigue JJ, Chow SC, Giannias B, Kubes P, Ferri LE (2012) Neutrophils Promote Liver Metastasis via Mac-1-Mediated Interactions with Circulating Tumor Cells. Cancer Res 72:3919-3927

93. Liang S, Fu C, Wagner D, Guo H, Zhan D, Dong C, Long M (2008) Two-dimensional kinetics of beta 2-integrin and ICAM-1 bindings between neutrophils and melanoma cells in a shear flow. Am J Physiol Cell Physiol 294:C743-C753

94. Liang S, Hoskins M, Khanna P, Kunz RF, Dong C (2008) Effects of the Tumor-Leukocyte Microenvironment on Melanoma-Neutrophil Adhesion to the Endothelium in a Shear Flow. Cell Mol Bioeng 1:189-200
95. Davis JC Jr, Manzi S, Yarboro C, Rairie J, McInnes I, Averthelyi D, Sinicropi D, Hale VG, Balow J, Austin H et al (1999) Recombinant human Dnase I (rhDNase) in patients with lupus nephritis. Lupus 8:68-76

96. Sugihara S, Yamamoto T, Tanaka H, Kambara T, Hiraoka T, Miyauchi Y (1993) Deoxyribonuclease treatment prevents blood-borne liver metastasis of cutaneously transplanted tumour cells in mice. Br J Cancer 67:66-70

97. Patutina O, Mironova N, Ryabchikova E, Popova N, Nikolin V, Kaledin V, Vlassov V, Zenkova M (2011) Inhibition of metastasis development by daily administration of ultralow doses of RNase A and DNase I. Biochimie 93:689-696

98. Foekens JA, Ries C, Look MP, Gippner-Steppert C, Klijn JG, Jochum M (2003) The prognostic value of polymorphonuclear leukocyte elastase in patients with primary breast cancer. Cancer Res 63:337-341

99. Nagamatsu Y, Iwasaki Y, Omura H, Hayashida R, Kashihara M, Nishi T, Yoshiyama K, Shirouzu K (2012) Neutrophil elastase activity in pulmonary venous blood during lung resection. Interact Cardiovasc Thorac Surg 15:452-455

100. Makino H, Kunisaki C, Kosaka T, Akiyama H, Morita S, Endo I (2011) Perioperative use of a neutrophil elastase inhibitor in video-assisted thoracoscopic oesophagectomy for cancer. Br J Surg 98:975-982

101. Iwahashi M, Nakamori M, Nakamura M, Ojima T, Naka T, Yamaue H (2011) Optimal period for the prophylactic administration of neutrophil elastase inhibitor for patients with esophageal cancer undergoing esophagectomy. World J Surg 35:1573-1579

102. Kawahara Y, Ninomiya I, Fujimura T, Funaki H, Nakagawara H, Takamura H, Oyama K, Tajima H, Fushida S, Inaba H et al (2010) Prospective randomized controlled study on the effects of perioperative administration of a neutrophil elastase inhibitor to patients undergoing video-assisted thoracoscopic surgery for thoracic esophageal cancer. Dis Esophagus 23:329-339

103. Kumar V, Sharma A (2010) Neutrophils: Cinderella of innate immune system. Int Immunopharmacol 10:1325-1334

104. Brandau S, Dumitru CA, Lang S (2013) Protumor and antitumor functions of neutrophil granulocytes. Semin Immunopathol 35(2):163-176

105. Papayannopoulos V, Staab D, Zychlinsky A (2011) Neutrophil elastase enhances sputum solubilization in cystic fibrosis patients receiving DNase therapy. PLoS One 6:e28526

106. Macdonald SJ, Dowle MD, Harrison LA, Shah P, Johnson MR, Inglis GG, Clarke GD, Smith RA, Humphreys D, Molloy CR et al (2001) The discovery of a potent, intracellular, orally bioavailable, long duration inhibitor of human neutrophil elastase-GW311616A a development candidate. Bioorg Med Chem Lett 11:895-898 